\title{
Formal Security Verification of the Station-to-Station based Cell-Attachment Procedure of LDACS
}

\author{
Nils Mäurer ${ }^{1}{ }^{\mathrm{a}}{ }^{\mathrm{a}}$, Christoph Gentsch ${ }^{2} \mathbb{1}^{\mathrm{b}}$, Thomas Gräupl ${ }^{1} \mathbb{1}^{\mathrm{c}}$ and Corinna Schmitt ${ }^{3} \mathbb{C}^{\mathrm{d}}$ \\ ${ }^{1}$ Institute of Communication and Navigation, German Aerospace Center (DLR), Wessling, Germany \\ ${ }^{2}$ Institute of Data Science, German Aerospace Center (DLR), Jena, Germany \\ ${ }^{3}$ Research Institute CODE, Universität der Bundeswehr, München, Germany \\ \{nils.maeurer, thomas.graeupl, christoph.gentsch\}@dlr.de, corinna.schmitt@unibw.de
}

Keywords: Cybersecurity, Authentication, Key Establishment, Symbolic Model, LDACS, Tamarin

Abstract: Aeronautical communications systems are currently undergoing a modernization process. Analogue legacy systems shall be replaced with modern digital alternatives, offering higher bandwidth, increasing capacity and paving the way for Unmanned Aeronautical Vehicles (UAVs). One modern candidate technology is the L-band Digital Aeronautical Communications System (LDACS), enabling long-range safety-critical digital communications between aircraft and ground. As with any modern wireless communications system, LDACS is prone to cyber-attacks. These issues were addressed in former research, where a secure cell-attachment procedure for LDACS, based on a modified Station to Station (STS) Mutual Authentication and Key Establishment (MAKE) protocol, was proposed. However, as of now, its security has not been proven. The contribution of this paper is the formal verification of the executability and security of the LDACS cell-attachment procedure using the symbolic model checker Tamarin. The achieved results proved that the suggested cell-attachment procedure for LDACS is workable and enables secure communication between aircraft and ground.

\section{Introduction}

One of the main pillars of the modern Air Traffic Management (ATM) system is a communication infrastructure that enables efficient aircraft control and safe separation in all phases of flight. Current communication systems are technically mature but suffering from the Very High Frequency (VHF) band's increasing saturation in high-density areas and the limitations posed by analogue radio communications. To overcome the capacity constraints of the legacy analogue systems, digitalization of aeronautical communications is necessary and currently underway (Mahmoud et al., 2014).

One of the candidate data link technologies for long-range terrestrial aeronautical communications is the L-band Digital Aeronautical Communications System (LDACS) (Schnell et al., 2014), which is a ground-based cellular digital aeronautical communications system for flight guidance and

\footnotetext{
a (iD) https://orcid.org/0000-0003-1324-7574

b (iD) https://orcid.org/0000-0001-7189-3465

c (i) https://orcid.org/0000-0002-7864-774X

d(D) https://orcid.org/0000-0002-4118-1878
}

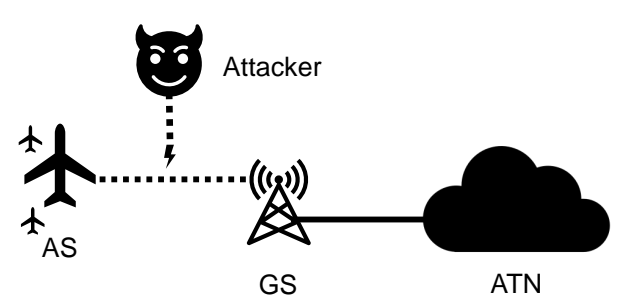

Figure 1: LDACS network architecture

communications related to the safety and regularity of flight. It is currently under standardization in the International Civil Aviation Organization (ICAO) (International Civil Aviation Organization (ICAO), 2018a) and has been tested in experimental flight trials (Mäurer et al., 2021a). Figure 1 illustrates the assumed network architecture. An Aircraft Station (AS) connects to a LDACS Ground Station (GS), the transmission site, which can serve several hundred ASs. The GS connects via the secure LDACS sub-network to the global Aeronautical Telecommunications Network (ATN). Without an authenticated key establishment, an active attacker can send arbitrary messages to both, AS and GS.

LDACS is foreseen to support a multitude of Air Traffic Services (ATS) and Aeronautical Operational 
Control (AOC) services, all related to the safety and regularity of flight (Gräupl et al., 2020). To guarantee this, cybersecurity is one of the key requirements of LDACS and a cybersecurity architecture has been proposed in (Mäurer, N. and Bilzhause, A., 2018). Core of this architecture is the LDACS cellattachment procedure, consisting of a cell entry procedure, where LDACS radios aboard the aircraft, establish contact with the ground counterpart, enabling basic communication. The second step of the cellattachment procedure is the Mutual Authentication and Key Establishment (MAKE) protocol, establishing trust and a shared secret between AS and GS (Mäurer, N. and Bilzhause, A., 2018). The MAKE protocol assumes an existing Public Key Infrastructure (PKI) with certificates set up on all authorized AS and GS, similar to the deployed airport communications system Aeronautical Mobile Airport Communication System (AeroMACS) (Crowe, 2016).

A comparison of scientific literature with official aeronautical standards of systems (Aeronautical Radio, Incorporated (ARINC), 2007; Blanchet, 2017) reveals, that after an aeronautical system is specified and deployed, necessary changes due to newly found security vulnerabilities are rarely applied. For example (Blanchet, 2017) analyzes the ACARS Secure Message (AMS) secure session initiation scheme using ProVerif, which is a symbolic model checker, and reveals a possible replay attack not addressed in the standard, yet. This is why a formal proof of security of the cell-attachment procedure is paramount, before the LDACS specification is finalized.

The objective of this paper is to provide a formal prove of the security of the cell-attachment procedure of LDACS by applying the symbolic model checker Tamarin (Meier et al., 2013).

\section{The LDACS Cell-Attachment Procedure}

We focus on the cell-attachment procedure, combining the cell entry procedure and certificate based Station to Station (STS)-MAKE protocol for LDACS as defined in (Mäurer, N. and Bilzhause, A., 2018) and updated in (Mäurer et al., 2021b).

\subsection{High Level Security Objectives}

In (Bilzhause et al., 2017) the authors originally identified five objectives for LDACS, which were later extended to nine in the LDACS Standards and Recommended Practises (SARPS) endorsed by ICAO (International Civil Aviation Organization (ICAO), 2018b). These objectives originated from previous threats- and risk analysis (Mäurer and Schmitt, 2019) of threats to ground- or aircraft stations. Within these works, concrete attack examples were given as, e.g., transmission of forged Automatic Dependent Surveillance-Contract (ADS-C) messages from aircraft to ground, hence falsifying the direction, position or velocity of an aircraft or the transmission of forged ATS or AOC messages, resulting in possibly dangerous flight instructions in digital voice or Controller-Pilot Data Link Communications (CPDLC) messages.

Attacks accomplishing these tasks are either to (a) falsify genuine messages, e.g., change the message content, sender or recipient, (b) to completely forge messages or to (c) repeat genuine messages unchanged, but at a point in time, they are not valid anymore. To combat such attacks, security controls such as authentication and integrity checks of messages via Message Authentication Codes (MAC) and sequence numbers and timestamps in messages must be put in place. However, to enable these message integrityand authenticity checks, trust has to be established between communication partners and the establishment of a shared session key between authenticated stations is required. Finally, the cell-attachment procedure needs to be executable, which is formulated as a provable lemma in Section 4.2.

\subsection{Security Objectives}

The radio link technology is the first point of contact between aircraft and ground (International Civil Aviation Organization (ICAO), 2015). Hence, the objective of the LDACS cell-attachment procedure is to establish mutual trust between any two parties AS and GS and a shared session key $K$ between any two parties AS and GS, in which they can have "mutual belief". Following the hierarchy of authentication and key establishment goals of Boyd et al. (Boyd et al., 2020), this mutual belief goal can be split up into the sub-goals entity authentication, key confirmation and good key, which we model via the lemmata Mutual Authentication, Secure Key Establishment and Perfect Forward Secrecy. Table 1 summarizes the attacks on the cell-attachment procedure, security controls defending against these attacks and the corresponding lemmata.

The objectives of the cell-attachment procedure can be summarized in the form of objectives and lemmata as follows:

O1* - Mutual Authentication: Both parties can be sure of the identity of the other and that both actually participated in this interaction. 
Table 1: LDACS MAKE protocol - Attacks \& Controls

\begin{tabular}{|l|l|l|}
\hline Attack & $\begin{array}{l}\text { Security } \\
\text { Requirement }\end{array}$ & Proof Lemma \\
\hline $\begin{array}{l}\text { Unknown key } \\
\text { share }\end{array}$ & $\begin{array}{l}\text { Each party shares the key } \\
\text { with the party it intended to }\end{array}$ & $\begin{array}{l}\text { Secure Key } \\
\text { Establishment, (Mutual) } \\
\text { Authentication }\end{array}$ \\
\hline $\begin{array}{l}\text { Man in the } \\
\text { middle }\end{array}$ & $\begin{array}{l}\text { Each party shares the key } \\
\text { with the party it intended to }\end{array}$ & $\begin{array}{l}\text { Secure Key } \\
\text { Establishment, (Mutual) } \\
\text { Authentication }\end{array}$ \\
\hline Eavesdropping & $\begin{array}{l}\text { The key is secret between } \\
\text { the two parties }\end{array}$ & $\begin{array}{l}\text { Secure Key } \\
\text { Establishment, (Perfect } \\
\text { Forward) Secrecy }\end{array}$ \\
\hline Replay & Key freshness & $\begin{array}{l}\text { Secure Key } \\
\text { Establishment }\end{array}$ \\
\hline $\begin{array}{l}\text { Long-term key } \\
\text { compromise }\end{array}$ & $\begin{array}{l}\text { Attacker can only authen- } \\
\text { ticate as the station, whose } \\
\text { keys were stolen }\end{array}$ & $\begin{array}{l}\text { (Mutual) } \\
\text { Authentication }\end{array}$ \\
\hline $\begin{array}{l}\text { Long-term key } \\
\text { compromise }\end{array}$ & $\begin{array}{l}\text { Old session keys of the } \\
\text { station must not be recon- } \\
\text { structable by the attacker }\end{array}$ & Perfect Forward Secrecy \\
\hline $\begin{array}{l}\text { Session key } \\
\text { leakage }\end{array}$ & $\begin{array}{l}\text { No other session keys (from } \\
\text { other stations or future } \\
\text { sessions) are affected }\end{array}$ & Perfect Forward Secrecy \\
\hline
\end{tabular}

02* - Secure Key Establishment: Both parties have established a shared session key, which means both parties know this key and know that they can use it for a secure communication with the other party for the duration of this session. The key must have never been used before in a session and only the two parties can know it.

O3* - Perfect Forward Secrecy: The established session key remains secret, even when the private signing keys of the involved parties have been compromised after this session, or other sessions keys have been leaked before.

In Section 4.2 these definitions are transformed into Tamarin provable symbolic lemmata.

\subsection{The LDACS Cell Entry Procedure}

The LDACS cell-attachment procedure begins with the cell entry procedure as depicted in Figure 2. It uses the logical Broadcast Control Channel (BCCH), Random Access Channel (RACH), Common Control Channel $(\mathrm{CCCH})$, and Data Channel (DCH) defined in (Gräupl et al., 2020).

Once a GS is securely connected to the aeronautical ground network, it starts sending a broadcast message called SIB, containing relevant information such as network identification, physical parameters such as channel frequencies and more. When an AS enters the cell served by that GS, it receives the broadcast message and sends a CELL_RQST message in reply. The CELL_RQST message contains a LDACS radio address. When the GS receives the CELL_RQST message, a CELL_RESP message is sent back to the AS, informing the AS about its local temporary address in the cell. After this exchange of control channel messages, both communication parties are connected and can start transmitting data in the DCH.

Note, that no cryptographic information has been

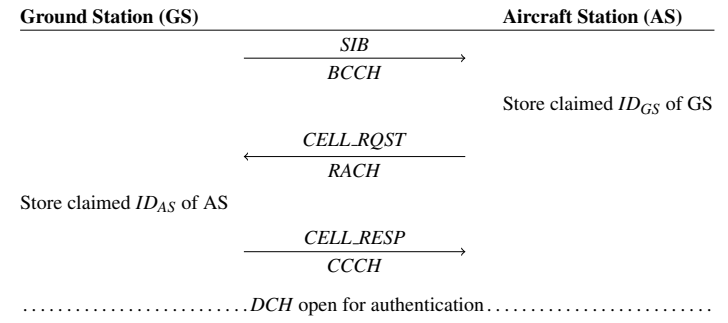

Figure 2: LDACS Cell Entry Procedure

exchanged, yet. This is because of the sizes of LDACS control channels: The $\mathrm{BCCH}$ allows for 1000 bits, the RACH 53 bits and the $\mathrm{CCCH}$ only for 728 bits maximum (Gräupl et al., 2020). However, since LDACS relevant parameters need to be exchanged here, the actual space to add cryptographic parameters is reduced even further. Hence, the MAKE protocol begins after the cell entry procedure using the user data channel DCH, allowing for different Diffie-Hellman key sizes and overall higher flexibility in transporting authentication data than over to the fixed-sized control channels.

\subsection{The LDACS MAKE protocol}

After the cell entry procedure, the LDACS MAKE protocol is performed over the DCH. Table 2 defines the used notation and Figure 3 illustrates the five steps of the realized protocol.

Table 2: Notations used in the MAKE protocol

\begin{tabular}{|c|c|}
\hline Notation & Definition \\
\hline \begin{tabular}{l|l} 
msg1 & msg2
\end{tabular} & Concatenation msg1 with msg2 \\
\hline$I D_{A}$ & Identifier of A \\
\hline PrivKey $_{A}$ & Private key in PKI of A \\
\hline $\mathrm{PubKey}_{A}$ & Public key in PKI of A \\
\hline $\operatorname{Sig}_{A}($ data $)$ & Signature of A with input data \\
\hline$x, y$ & Ephemeral private key of entity A, B \\
\hline$t_{A}$ & Ephemeral public key of entity A \\
\hline$g$ & Public Diffie-Hellman parameters \\
\hline$S_{A, B}$ & Shared Diffie-Hellman key of A, B \\
\hline$K D F$ & Key Derivation Function \\
\hline$K_{A, B}$ & Session key of A, B \\
\hline$N_{A}$ & Nonce of entity A \\
\hline
\end{tabular}

In (Mäurer et al., 2021b) sets of cryptographic algorithms for the tasks of signing, encrypting or authenticating messages were proposed. However, since this work focuses on the symbolic model of the MAKE protocol, specific cryptographic algorithms are not assigned here. 


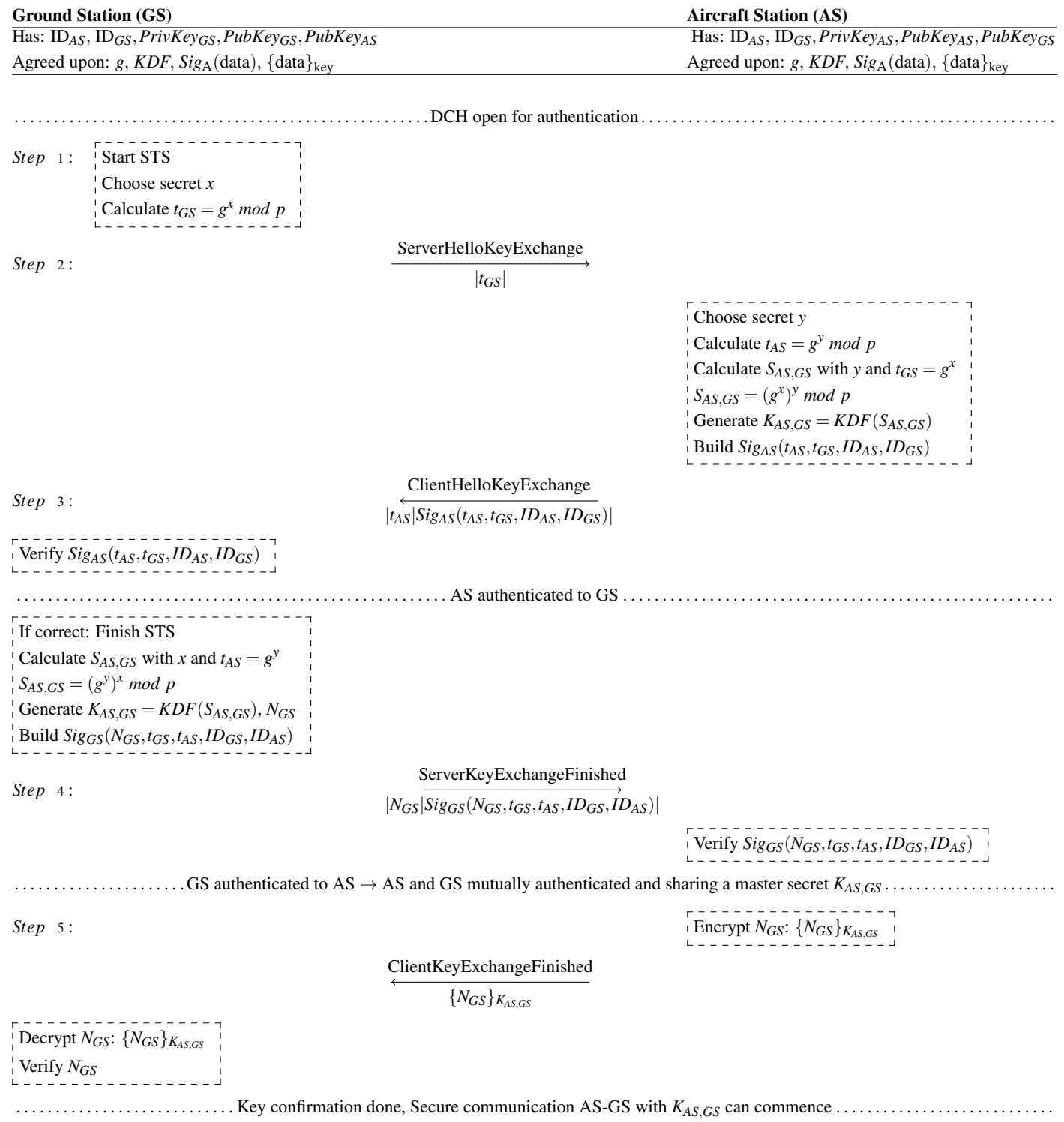

Figure 3: LDACS STS-MAKE Protocol

\section{Tamarin Model of the LDACS Cell-Attachment Procedure}

Mechanized protocol verification tools rely either on the (1) symbolic or the (2) computational model. In the first case, the Dolev-Yao model (Dolev and Yao, 1981), cryptographic primitives are black boxes, which are "unbreakable" as long as the attacker has no possession of the right key. Messages are terms of these primitives and the adversary can only apply these primitives. This is in contrast to the computational model (Yao, 1982), where messages are regarded as bitstrings and cryptographic primitives are modeled as functions on these bitstrings. The adver- sary is regarded as a probabilistic Turing machine. "Although in some restricted cases a proof in the Dolev-Yao model can imply a computational proof [...], this is in general not the case" (Boyd et al., 2020). Therefore, only the symbolic model is considered applying Tamarin (Meier et al., 2013) in the performed analysis.

\subsection{Modeling Notes}

The standards from the Tamarin documentation (Basin et al., 2021) are followed while modeling the cell-attachment procedure. As there is no builtin role type in Tamarin, only rules and facts, roles must be modeled using state facts, linking multi- 
ple rules together by carrying the state of a role from rule to rule. This way, the roles of AS and GS are modeled.

Roles, Instances and Sessions: In the present model of the roles 'AS' and 'GS', it is distinguished between two levels of 'identity': Each role can be instantiated infinitely, identified by the public instancevariables $\$ A S$ and $\$ G S$. This reflects the fact, that in the real world there can be many aircraft and groundstations. Each instance of an aircraft or a groundstation needs a public-key pair to participate in a protocol run. In the presented model, the existence of a global PKI is assumed, where every principal has exactly one such public-key pair. This is reflected in the developed and applied Tamarin model by the first rule (cf. Listing 1), which enables any $\$ X$ to register a key-pair only once.

This is modeled via the persistent fact $! \operatorname{Ltk}(\$ X$ , ltkX), which stores the private long-term key $l t k X$ in the global key-store; and the fact! $P k(\$ X, p k(l t k X))$, that stores the corresponding public key of $\$ X$ respectively. Finally the public key of $\$ X$ is also sent to the attacker by $\operatorname{Out}(p k(l t k X))$.

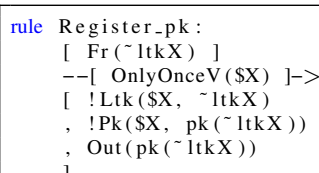

Listing 1: Tamarin "Register_pk" rule

In the applied model, each role can participate in an unlimited number of protocol runs. This is called "sessions", and they are modeled by creating a fresh session-ID at the beginning of each instance, which will be used by the instance until the end of the protocol run and helps to distinguish multiple sessions of one instance in the performed proof. Both roles start with the minimal knowledge of their own ID and their own long-term key. They get to know each other the first time, when the AS receives the broadcast message of the GS, advertising its own ID - answered by the AS with a "CellEntryRequest", accompanied with its ID.

\subsection{Security Assumptions}

In the model presented in this paper, the following assumptions about the building blocks in the LDACS cell-attachment procedure are made: The modeled cryptosystems are treated as black boxes meaning that (1) signature and (2) encryption mechanisms are assumed secure as long as the appropriate keys are unknown to the adversary. That way, it is assumed, (3) that the adversary cannot learn anything from messages he or she cannot decrypt. Also, (4) a guaranteed freshness is assumed for all generated values like session IDs or nonces. Physical properties of the communication - e.g. timing-issues of the message exchange - are not modeled and therefore (5) side channel attacks are not captured. A feature of Tamarin is (6) the unbound number of stations and therefore parallel or interleaved protocol runs by default, which allows to identify the most complex attacks on the protocol.

Certificates: It is foreseen, that AS and GS have certificates (handled by an LDACS PKI), signed by a trusted Certificate Authority (CA) proving their identity claim. AS and GS have all necessary public keys of respective communication partners pre-installed, to verify signatures and identity claims. Furthermore the signatures used in certificates are unforgeable under chosen-plaintext attacks (Goldwasser et al., 1988).

Signatures: Long term key pairs of AS and GS are used for signatures only and signatures are assumed to be strongly unforgeable under chosenplaintext attacks (Goldwasser et al., 1988).

Diffie-Hellman: Shared Diffie-Hellman parameters and the choice of Diffie-Hellman Key Exchange (DHKE) variation were agreed upon, prior to the MAKE protocol. AS and GS generate a fresh public Diffie-Hellman key for each protocol run (i.e., $t_{A S}$, $\left.t_{G S}\right)$. Hence, $t_{A S}, t_{G S}$ can also serve as nonces, assuming enough entropy in the underlying random number generator for secret $x$ and $y$. Finally, independent of the choice of chosen DHKE procedure for LDACS (Mäurer et al., 2021b), the underlying Diffie-Hellman assumption is assumed to hold.

Key Derivation: It is assumed that the Key Derivation Function (KDF) is able to derive an arbitrary amount of strong cryptographic keys from a usually not uniformly distributed input source and define "strong cryptographic keys" as "indistinguishable from a random uniform string of the same length" (Krawczyk, 2010).

\section{Symbolic Security Proof}

The defined security objectives for the cellattachment procedure in Section 2.2 are proven with the Tamarin model presented in section 3.

\subsection{Attacker Model}

Tamarin follows the approach of Dolev-Yao (Dolev and Yao, 1981), where an ideal, powerful attacker is assumed. Additionally, the possibility is as- 
sumed that the adversary can corrupt any station and obtain their long-term secret (certificate), or their session keys used in different runs of the protocol. As the standard Dolev-Yao-Attacker is already built-in to Tamarin, only the Tamarin rules for the key compromise of long-term and session-keys have to be added.

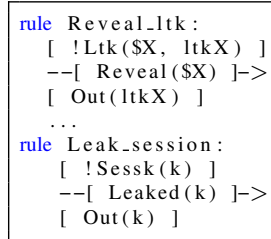

Listing 2: Tamarin rules for key compromise of long-term and session-keys

These are given in Listing 2 and enable the attacker to corrupt any agent in the model by default.

\subsection{Tamarin Lemmata}

In addition to the executability of the LDACS cellattachment procedure, the security objectives $\mathrm{O} 1 *$ to O3* of Section 2.2 have to be proven, as well. All these objectives are formalized by their appropriate Tamarin lemma in the following.

Lemma 1: "Executable" (cf. Listing 3): There exists a trace where instance A in role AS participates in session $i_{a}$ and instance $\mathrm{B}$ in role GS participates in session $i_{b}$, A is requesting $\mathrm{B}$ for cell entry, both are starting the protocol by exchanging $t_{A S}$ and $t_{G S}$, and finally both commit by having the same shared data.

Lemma 2: "Mutual Authentication via Injective Agreement" (cf. Listing 4): If A finishes a run with $\mathrm{B}$ by exchanging $\mathrm{y}$, it can be sure, $\mathrm{B}$ also ran the protocol with A and y has not been exchanged before in any other run. Only exception: the private key of an honest agent has been compromised before.

Lemma 3: "Secure Key Establishment" (cf. Listing 5): If $\mathrm{A}$ finishes a run with $\mathrm{B}$, it can be sure, that it has a fresh key $\mathrm{P}$ and that $\mathrm{B}$ also has this key for use with A, and this key has not been established before, implicating that also no other agent knows it. Only exception: the private key of an honest agent has been corrupted before.

Lemma 4: "Perfect Forward Secrecy" (cf. Listing 6): The exchanged session key $\left(K_{A S, G S}\right)$ cannot be known by the attacker, even when he acquires the private key of one or both parties later on. The case, the session key was leaked to the attacker, is excluded.

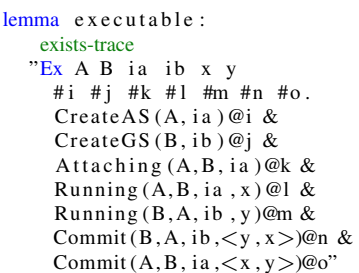

Listing 3: Lemma 1

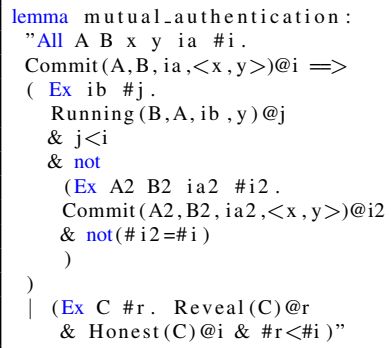

Listing 4: Lemma 2

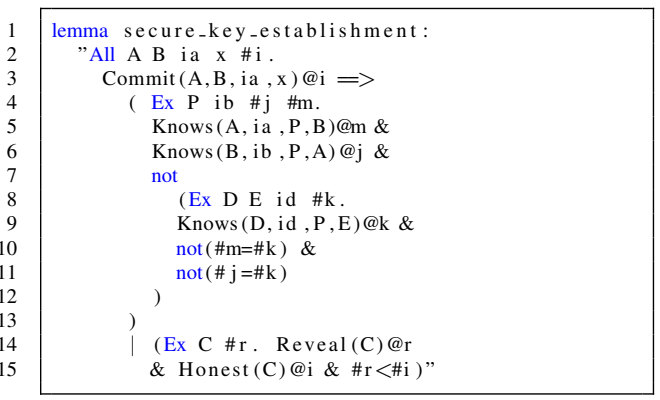

Listing 5: Lemma 3

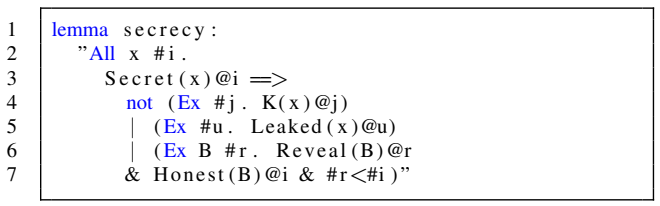

Listing 6: Lemma 4

\subsection{Result}

For evaluation purposes the Tamarin prover version 1.6.0 in automatic mode was used to prove the four lemmata presented in Section 4.2. The verification took 36.159 s on a Ubuntu 18.04 Laptop with an Intel(R) Core(TM) i7-8650U CPU and 16GB of RAM. All four lemmata could be verified without interaction. The source code of the Tamarin model is available for download at GitHub ${ }^{1}$. In Table 3 the Tamarin

\footnotetext{
${ }^{1}$ https://github.com/kr4ck-com/LDACS-MAKE, accessed May 18, 2021
} 
output for each lemma is presented. The scope column states which type of proof has been done: 'existstrace'-proofs verify, that the given property or lemma holds at least for one trace of the protocol; 'all-traces'proofs respectively verify that the property holds for all traces. The last column gives the number of verification steps that were executed by Tamarin to verify the lemma. As all lemmata have been proven to

Table 3: Tamarin verification results

\begin{tabular}{|l|c|c|r|}
\hline Lemma & Scope & Result & Steps \\
\hline \hline Executable & Exists-trace & $\boldsymbol{V}_{\text {Verified }}$ & 20 \\
\hline $\begin{array}{l}\text { Mutual Authen- } \\
\text { tication }\left(\mathrm{O}{ }^{*}\right)\end{array}$ & All-traces & $\boldsymbol{V}_{\text {Verified }}$ & 29 \\
\hline $\begin{array}{l}\text { Secure Key } \\
\text { Exchange (O2*) }\end{array}$ & All-traces & $\boldsymbol{V}_{\text {Verified }}$ & 1158 \\
\hline $\begin{array}{l}\text { Perfect Forward } \\
\text { Secrecy (O3*) }\end{array}$ & All-traces & $\boldsymbol{V}_{\text {Verified }}$ & 43 \\
\hline
\end{tabular}

hold, all required security controls of the LDACS cellattachment procedure defined in Table 3 also hold.

\section{Discussion and Conclusions}

The achieved results show that the LDACS attachment procedure, consisting of cell entry procedure and modified STS MAKE protocol is secure in fulfilling (1) mutual authentication, (2) secure key agreement (3) perfect forward secrecy for subsequent key material, (4) consistency, and (5) executability. The presented proof is valid only under the assumptions of Section 3.2 and there are limitations originating from the method of symbolic model checking.

As indicated in Section 3.2 there is no guarantee for computational soundness of the underlying cryptographic algorithms of the protocol. E.g., in the symbolic model, the "Diffie-Hellman-assumption" cannot be verified or falsified, hence in the context of the prove it is assumed to hold. The same holds true for the other used cryptographic primitives as signatures or encryption schemes. Also, any assertions regarding possible vulnerabilities of an actual software implementation of the protocol, e.g., because of buffer overflows, cannot be made. Also, side-channel attacks because of e.g. timing issues cannot be found in this way, since they are also implementation-specific. Another point is that the current LDACS cell-attachment procedure, in the way it is presented in this paper, has no denial of service protection, which is solved on protocol level for e.g., fail-stop protocols with cookies. This needs to be investigated further.

Many of the assumptions from Section 3.2 rely on the secure setup and operation of the underly- ing LDACS PKI. For instance, the assumed impossibility of unauthorized key registration with a spoofed ID, the privacy of private signing keys, storage limits and an autonomous registration of station public keys are all dependent on the way the PKI is set up and how keys are transported. In addition, similarities between the LDACS PKI and AeroMACS PKI (Crowe, 2016) are assumed. As this is a requirement for the proposed LDACS cellattachment procedure to work as intended, due to the corroboration of identity and public keys and trust established within the chain-of-trust model that the PKI uses, it is reasonable to assume such a PKI to be built for LDACS. However, already the second assumption of a trustworthy CA, proves difficult in practice, with many examples where intermediate sub-CAs were compromised in the past (Roosa and Schultze, 2013). As there is no such incident reported for the AeroMACS PKI as of yet and with ICAO's efforts to establish their own aviation PKI (Patel, 2016), the underlying PKI infrastructure will likely become more robust in the future.

The contribution of this paper is the formal proof of the security of the LDACS cell-attachement procedure. Using the symbolic model checker Tamarin, a mathematical, formal model of this procedure was built, focusing on the cryptographic aspects of the MAKE protocol. Tamarin proved that the cellattachment is secure in the symbolic model and is proven to have no design flaws in its architecture. This constitutes an important step for the development of the general LDACS cybersecurity architecture since authentication and key establishment are the most crucial steps in establishing secure wireless communication. For future research, the investigation of the agreement procedure for Diffie-Hellman parameters, as well as control channel security for the control channels of LDACS, are interesting, open tasks.

\section{REFERENCES}

Aeronautical Radio, Incorporated (ARINC) (2007). DATALINK SECURITY PART 1 - ACARS MESSAGE SECURITY. Technical report, Aeronautical Radio, Incorporated (ARINC).

Basin, D., Cremers, C., Dreier, J., Meier, S., Sasse, R., and Schmidt, B. (2020 (accessed May 18, 2021)). Tamarin Prover Manual.

Bilzhause, A., Belgacem, B., Mostafa, M., and Gräupl, T. (2017). Datalink Security In The L-band Digital Aeronautical Communications System (LDACS) For Air Traffic Management. Aerospace and Electronic Systems Magazine, 32(11):22-33.

Blanchet, B. (2017). Symbolic And Computational Mechanized Verification Of The ARINC823 Avionic Proto- 
cols. In 30th Computer Security Foundations Symposium (CSF), pages 68-82. IEEE.

Boyd, C., Mathuria, A., and Stebila, D. (2020). Protocols For Authentication And Key Establishment. Springer.

Crowe, B. (2016). Proposed AeroMACS PKI Specification Is A Model For Global And National Aeronautical PKI Deployments. In WiMAX Forum at 16th Integrated Communications, Navigation and Surveillance Conference (ICNS), pages 1-19. IEEE.

Dolev, D. and Yao, A. (1981). On The Security Of Public Key Protocols (Extended Abstract). In 22nd Annual Symposium on Foundations of Computer Science, pages 350-357. IEEE Computer Society.

Goldwasser, S., Micali, S., and Rivest, R. (1988). A Digital Signature Scheme Secure Against Adaptive Chosen-Message Attacks. SIAM Journal on computing, 17(2):281-308.

Gräupl, T., Rihacek, C., and Haindl, B. (2020). LDACS A/G Specification. SESAR2020 PJ14-02-01 D3.3.030, German Aerospace Center (DLR).

International Civil Aviation Organization (ICAO) (2015). Doc 9896 - Manual On The Aeronautical Telecommunication Network (ATN) Using Internet Protocol Suite (IPS) Standards And Protocols. Technical report, International Civil Aviation Organization (ICAO).

International Civil Aviation Organization (ICAO) (2018a). Finalization of LDACS Draft SARPs - Working Paper WP05 including Appendix. Technical report, International Civil Aviation Organization (ICAO), Montreal, Canada.

International Civil Aviation Organization (ICAO) (2018b). Finalization Of LDACS Draft SARPs - Working Paper WP05 Including Appendix. Technical report, International Civil Aviation Organization (ICAO).

Krawczyk, H. (2010). Cryptographic Extraction And Key Derivation: The HKDF Scheme. In Annual Cryptology Conference, pages 631-648. Springer.

Mahmoud, M. S. B., Pirovano, A., and Larrieu, N. (2014). Aeronautical Communication Transition From Analog To Digital Data: A Network Security Survey. Computer Science Review, 11-12:1-29.

Mäurer, N., Gräupl, T., Bellido-Manganell, M., Mielke, D., Filip-Dhaubhadel, A., Heirich, O., Gerbeth, D., Felux, M., Schalk, L., Becker, D., Schneckenburger, N., and Schnell, M. (2021a). Flight Trial Demonstration of Secure GBAS via the L-band Digital Aeronautical Communications System (LDACS). IEEE Aerospace and Electronic Systems Magazine, pages 8-17.

Mäurer, N., Gräupl, T., and Schmitt, C. (2021b). Cybersecurity For The L-band Digital Aeronautical Communications System (LDACS). In Song, H., Hopkinson, K., Cola, T. d., Alexandrovich, T., and D., L., editors, Aviation Cybersecurity: Foundations, Principles, and Applications, pages 1-38. Institution of Engineering and Technology (IET).

Mäurer, N. and Schmitt, C. (2019). Towards Successful Realization Of The LDACS Cybersecurity Architecture: An Updated Datalink Security Threat- and Risk Analysis. In 19th Integrated Communications, Navigation and Surveillance Conference (ICNS), pages 1A2/11A2-13. IEEE.

Mäurer, N. and Bilzhause, A. (2018). A Cybersecurity Architecture For The L-band Digital Aeronautical Communications System (LDACS). In 37th Digital Avionics Systems Conference (DASC), pages 1-10. IEEE.

Meier, S., Schmidt, B., Cremers, C., and Basin, D. (2013). The TAMARIN Prover For The Symbolic Analysis Of Security Protocols. In 25th International Conference on Computer Aided Verification (CAV), page 696-701. Springer.

Patel, V. (2016). ICAO Air-Ground Security Standards Status ICNS Conference 2016. In Integrated Communications Navigation and Surveillance (ICNS), pages 1-31. IEEE.

Roosa, S. and Schultze, S. (2013). Trust Darknet: Control And Compromise In The Internet's Certificate Authority Model. IEEE Internet Computing, 17(3):1825.

Schnell, M., Epple, U., Shutin, D., and Schneckenburger, N. (2014). LDACS: Future Aeronautical Communications For Air-Traffic Management. IEEE Communications Magazine, 52(5):104-110.

Yao, A. (1982). Theory And Application Of Trapdoor Functions. In 23rd Annual Symposium on Foundations of Computer Science (SFCS), pages 80-91. IEEE.

\section{APPENDIX}

AeroMACS Aeronautical Mobile Airport Communication System

AOC Aeronautical Operational Control

AS Aircraft Station

ATN Aeronautical Telecommunications Network

ATS Air Traffic Services

BCCH Broadcast Control Channel

CCCH Common Control Channel

CPDLC Controller-Pilot Data Link

Communications

DCH Data Channel

DHKE Diffie-Hellman Key Exchange

GS Ground Station

ICAO International Civil Aviation Organization

KDF Key Derivation Function

LDACS L-band Digital Aeronautical Communications System

MAKE Mutual Authentication and Key Establishment

PKI Public Key Infrastructure

RACH Random Access Channel

STS Station to Station 